\title{
Activin A expression in esophageal carcinoma and its association with tumor aggressiveness and differentiation
}

\author{
ZHENHUA WANG ${ }^{1}$, NING ZHANG $^{2}$, RUIFENG SONG ${ }^{3}$, RUITAI FAN $^{4}$, LIUQIN YANG $^{1}$ and LIPING WU ${ }^{1}$ \\ Departments of ${ }^{1}$ Radiotherapy and ${ }^{2}$ Neurology, Xinxiang Central Hospital, Xinxiang, Henan 453000; \\ Departments of ${ }^{3}$ Gastroenterology and ${ }^{4}$ Radiotherapy, The First Affiliated Hospital of Zhengzhou University, \\ Zhengzhou, Henan 450052, P.R. China
}

Received September 20, 2014; Accepted May 8, 2015

DOI: $10.3892 / \mathrm{ol} .2015 .3248$

\begin{abstract}
The aim of the present study was to investigate the expression of activin A in esophageal carcinoma and its association with tumor differentiation and metastasis. A total of 57 esophageal carcinoma patients and 36 controls were included in the current study. The mRNA and protein expression levels of activin $\mathrm{A}$ in esophageal tumors or normal esophageal tissues were determined by reverse transcription-quantitative polymerase chain reaction and immunohistochemical analysis. In addition, the association of activin A expression with esophageal carcinoma differentiation, metastasis and recurrence postoperatively was analyzed. The mRNA and protein expression levels of activin $\mathrm{A}$ in esophageal carcinoma were significantly higher compared with those in normal esophageal tissues $(\mathrm{P}<0.05)$. The expression of activin A was higher in poorly-/moderately-differentiated esophageal tumor tissues compared with that of well-differentiated or control tissues $(\mathrm{P}<0.05)$. Furthermore, the expression of activin $\mathrm{A}$ in poorly-differentiated esophageal tumor tissues was higher compared with that of moderately-differentiated tissues $(\mathrm{P}<0.05)$. A positive correlation was also observed between differentiation degree and activin A expression. The expression of activin A was higher in patients with lymph node metastasis compared with those without metastasis $(\mathrm{P}<0.05)$. The cumulative survival rate of patients with a high expression of activin $A$ at 1,2 and 3 years postoperatively was significantly decreased compared with that of patients with a lower expression of activin $\mathrm{A}(\mathrm{P}<0.05)$; by contrast, the cumulative recurrence rate was significantly higher in patients with a lower activin A expression $(\mathrm{P}<0.05)$. In conclusion, abnormal expression of activin A was detected in esophageal tumor tissues, which was correlated with the
\end{abstract}

Correspondence to: Dr Zhenhua Wang, Department of Radiotherapy, Xinxiang Central Hospital, 56 Jinsui Road, Xinxiang, Henan 453000, P.R. China

E-mail: zhenhuawangcn@163.com

Key words: activin A, esophageal carcinoma, tumor aggressiveness, cell differentiation tumor differentiation, metastasis, survival and recurrence. In conclusion, activin A may be used as an auxiliary index in the diagnosis and prognosis of clinical esophageal carcinoma.

\section{Introduction}

Esophageal cancer is one of the most common malignancies, its incidence rate ranks eighth among cancers worldwide (1). The incidence rates vary widely between countries, with approximately half of all the diagnosed cases occurring in China (2). In addition, esophageal cancer is $\sim 3$ times more common in men than in women (3). The majority of patients are diagnosed with advanced stage disease, since the early symptoms are rarely detected. Systemic metastasis is the major cause of mortality in patients with esophageal cancer $(4,5)$. Therefore, developing novel methods for the early diagnosis of the disease is pivotal for the treatment of esophageal carcinoma patients; however, there is a lack of molecular targets for the diagnosis of esophageal cancer in clinical practice.

Activin A, which is a member of the transforming growth factor $\beta$ superfamily, was initially isolated from the porcine pituitary follicle and has the ability to induce differentiation of embryonic stem cells (6). There are three types of receptors for activin A: type I, II and III. Activin A binds to the type II receptor with the aid of the type III receptor, while it can cause the phosphorylation of type I receptor and then form a ternary complex with the phosphorylated type I receptor (7). Smad 2 and Smad3 in the cytosol can be phosphorylated by this complex and subsequently transferred into the nucleus, regulating the expression of certain genes (8-11). Activin A plays an important role in cell proliferation, differentiation, apoptosis and adhesion (12-14). Recent studies have demonstrated that activin A expression is significantly increased in various types of cancer and is correlated with cancer progression and metastasis (15-17). However, to the best of our knowledge no studies have previously investigated the expression of activin A in esophageal cancer tissues. In particular, the association of activin A with esophageal carcinoma differentiation and metastasis, as well as the survival and recurrence rates in esophageal carcinoma patients, remain to be elucidated.

In present study, the expression of activin A in esophageal cancer tissues was detected and its association with tumor differentiation, metastasis, postoperative survival and recurrence was 
analyzed, which may provide evidence for the development of novel clinical diagnosis methods for esophageal cancer.

\section{Patients and methods}

Patients. A total of 57 esophageal cancer tissue specimens were included in the study, which had been resected from patients receiving surgery at Xinxiang Central Hospital (Xinxiang, China), between June 2010 and June 2013. All the samples were pathologically diagnosed as esophageal carcinomas and termed the observation group. The 57 patients included 36 males and 21 females, with an age range of 44-67 years and mean age of $50.4 \pm 10.4$ years. In total, 17 tumor tissue specimens were well-differentiated, 25 were moderately-differentiated and 15 were poorly-differentiated. Lymph node metastasis was observed in 37 patients, while the other 20 patients presented no metastasis. All these patients were followed-up for 3 years after surgery. In addition, 36 esophageal tissue samples from individuals who underwent physical examination but did not present any pathological changes were selected as the control group. The 36 control patients included 25 males and 11 females, with an age range of 42-69 years and mean ages of $51.7 \pm 11.1$ years. The two groups demonstrated no statistically significant differences in their age, gender and other indicators $(\mathrm{P}>0.05)$. This study was conducted in accordance with the Declaration of Helsinki (The Seventh Revision, 2013) and with approval from the Ethics Committee of Xinxiang Central Hospital (Xinxiang, China). Written informed consent was obtained from all participants.

Reverse transcription-quantitative polymerase chain reaction (RT-qPCR). The esophageal tumor tissues or normal esophageal tissues were homogenized in $1 \mathrm{ml}$ TRIzol (Invitrogen Life Technologies, Carlsbad, CA, USA), and then $200 \mu 1$ chloroform was added and mixed. The mixture was naturally stratified on ice and then centrifuge at $15,000 \mathrm{x} \mathrm{g}$ for $15 \mathrm{~min}$, and $500 \mu \mathrm{l}$ of the supernatant was transferred and mixed with an equal volume of isopropanol. Next, the mixture was placed on ice for $15 \mathrm{~min}$, followed by collection of total RNA by centrifugation at $15,000 \mathrm{x} \mathrm{g}$ for $10 \mathrm{~min}$. The mixture was then washed twice with $75 \%$ cooling ethanol, and the precipitation was redissolved in diethylpyrocarbonate-treated sterilized water. Total RNA was quantified and reverse-transcribed into cDNA using an M-MLV Reverse Transcription kit. (Takara Biotechnology Co., Ltd., Dalian, China), and then used for qPCR.

Primers for qPCR were designed according to the mRNA sequence from GenBank (accession no. AY578797.1) using Primer Premier software, version 5.0 (Premier Biosoft, Palo Alto, CA, USA). The primers used were as follows: activin A forward, 5'-TTCTCGCTGTACTGCTGCAGA-3', and reverse, 5'-CTTCCTGCATGTCTTCAAGAGATG-3'; $\beta$-actin (internal control) forward, 5'-GCGGGAAATCGTGCGTGAC-3', and reverse, 5'-CGTCATACTCCTGCTTGCTG-3'. The reaction mixture was prepared with SYBR Green Master Mix (Takara Biotechnology Co.,Ltd.), $10 \mu \mathrm{mmol} / 1$ of each primer and $20-50 \mu \mathrm{g}$ cDNA, with a final volume of $20 \mu \mathrm{l}$. qPCR was performed under the following cycling parameters: $30 \mathrm{sec}$ at $95^{\circ} \mathrm{C}$, followed by 40 cycles of $3 \mathrm{sec}$ at $95^{\circ} \mathrm{C}$ and $30 \mathrm{sec}$ at $60^{\circ} \mathrm{C}$. The specificity of the product was determined by melting curve analysis. Data acquired were analyzed using the $2^{-\Delta \Delta \mathrm{Ct}}$ method (18).
Immunohistochemical assays. The tissue samples were embedded in paraffin and cut into $5-\mu \mathrm{m}$ sections, then mounted on polylysine-treated glass slides and dried for $1 \mathrm{~h}$ at $50^{\circ} \mathrm{C}$. Next, the slides were deparaffinized in xylene, rehydrated by graded ethanol, washed three times with distilled water and then heated for $8 \mathrm{~min}$ in sodium citrate solution. Subsequent to washing three times in phosphate-buffered saline (PBS), the tissues were quenched in $3 \% \mathrm{H}_{2} \mathrm{O}_{2}$ in methanol and then washed three times in PBS for 5 min each time. The tissues were blocked with PBS-Tween 20 containing 10\% goat serum for $30 \mathrm{~min}$ at $37^{\circ} \mathrm{C}$ and then incubated with mouse anti-human activin A antibody (1:100; sc-35644; Santa Cruz Biotechnology, Inc., Santa Cruz, CA, USA) at $4^{\circ} \mathrm{C}$ overnight. After washing three times in PBS to remove excess antibodies, the slides were incubated with diluted goat anti-mouse antibody (1:100; sc-38462; Santa Cruz Biotechnology, Inc.) for $30 \mathrm{~min}$ at $37^{\circ} \mathrm{C}$. Subsequent to washing for a further three times, $50 \mu \mathrm{l}$ of 3,3'-diaminobenzidine was used as the chromogen. Finally, the slides were rinsed with water, dehydrated in ethanol gradient and xylene, and then mounted using neutral resin (Dingguo Inc., Beijing, China).

Observation indexes and evaluation criteria. The mRNA and protein expression levels of activin A in the two groups (esophageal carcinoma or normal tissues) were observed. The expression levels of activin A in esophageal carcinoma patients with different degrees of differentiation and metastasis status were analyzed, as well as their association with the survival and tumor recurrence of patients. Tissue samples with $>50 \%$ activin A-positive cells in the immunohistochemical assay were defined as activin A-positive patients, while samples with $<50 \%$ positive cells were defined as activin A-negative patients.

Statistical analysis. The data are expressed as the mean \pm standard deviation and analyzed using the SPSS version 13.0 software (SPSS, Inc., Chicago, IL, USA). Measurement data was performed using Student's t-test. Comparisons of cumulative recurrence rate or cumulative survival rate were performed using the Kaplan-Meier method and log-rank test, respectively. $\mathrm{P}<0.05$ was considered to indicate a statistically significant difference.

\section{Results}

mRNA expression levels of activin A in esophageal carcinoma tissues. The mRNA level of activin A was detected using the RT-qPCR method. As shown in Fig. 1, the primers used for activin A were specific to the activin A gene and the melting curve was a simplex. In esophageal carcinoma tissues, the mRNA expression of activin A was significantly increased when compared with that in the normal esophageal tissues, and the difference was statistically significant $(\mathrm{P}<0.05)$.

Protein expression of activin A in esophageal carcinoma tissues. As shown in Fig. 2, activin A was expressed mainly in the cytoplasm, which appeared as brown staining in the immunohistochemical assay. A low expression of activin A was observed in the normal esophageal tissues, while the expression was evidently increased in esophageal carcinoma tissues. Quantitative analysis revealed that activin A expression in 
A

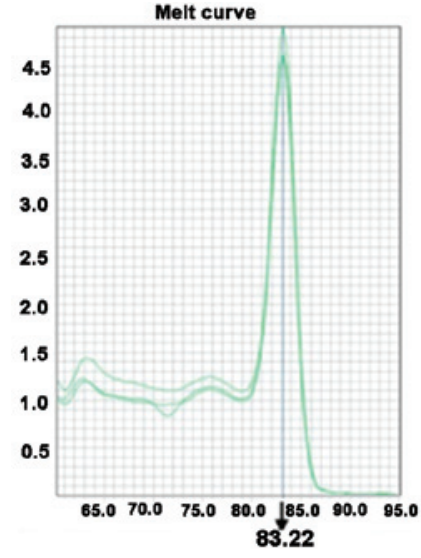

B

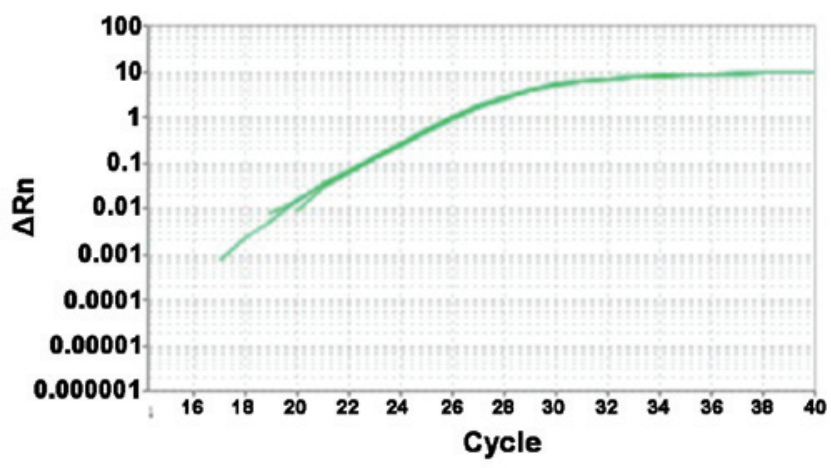

C

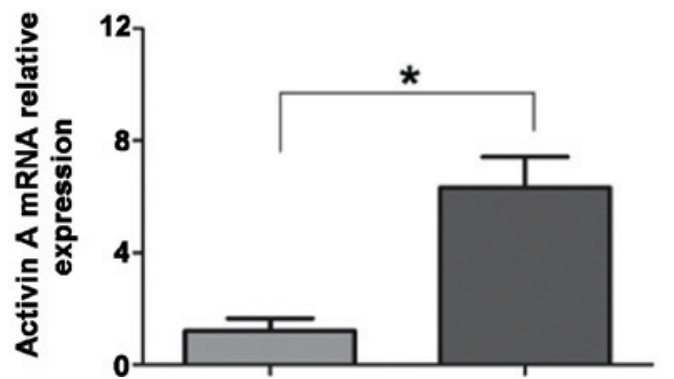

$\square$ Control group

$\square$ observation group

Figure 1. Comparison of mRNA expression levels of activin A in esophageal cancer and normal esophageal tissues. (A) Melting curve of activin A. (B) Amplification curve of activin A. (C) Analysis of expression levels of activin A in the observation (cancer) and control (normal tissues) groups. "P<0.05.

A

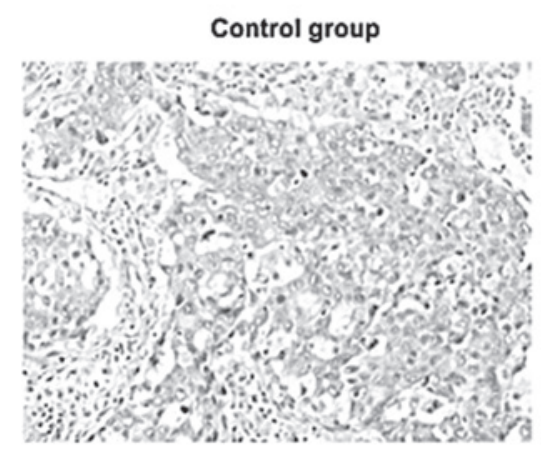

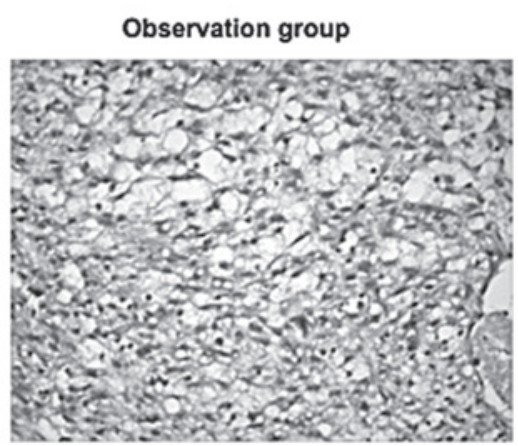

B

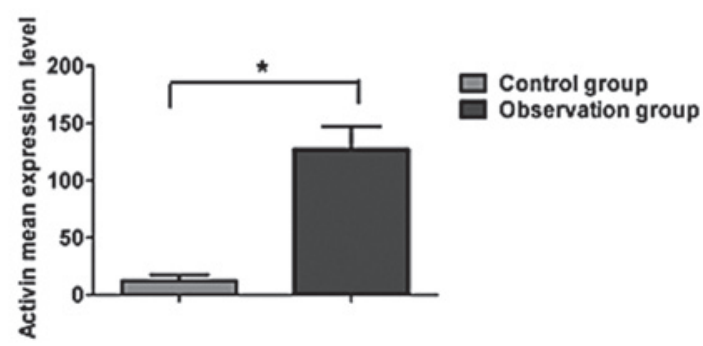

Figure 2. Comparison of protein expression levels of activin A in esophageal cancer and normal esophageal tissues. (A) Immunohistochemical analysis and (B) quantitative analysis, revealing increased activin A expression in the observation group (esophageal cancer tissues), compared with the control group (normal esophageal squamous tissues).

esophageal cancer was significantly higher compared with that in normal esophageal tissues, and the difference was statistically significant $(\mathrm{P}<0.05)$.

Expression levels of activin A in esophageal carcinoma tissues with various differentiation degrees. As shown in
Fig. 3, the expression of activin A was relatively low in well-differentiated esophageal carcinoma tissues, higher in moderately-differentiated esophageal carcinoma tissues and the highest in poorly-differentiated tumors. Quantitative analysis demonstrated that the expression of activin A was significantly higher in poorly- and 
A

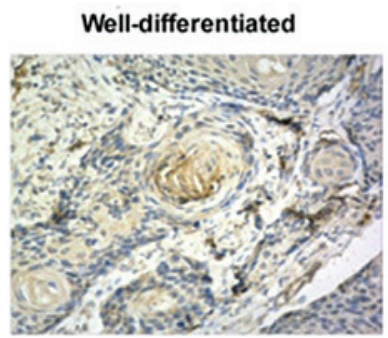

Moderately differentiated

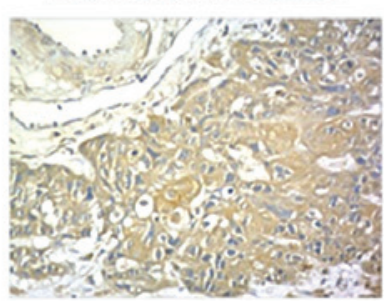

Poorly differentiated

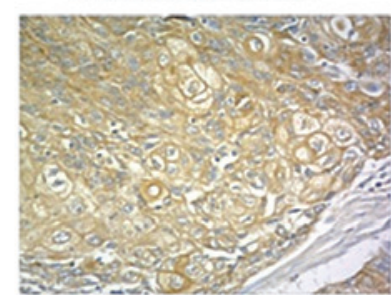

Control group

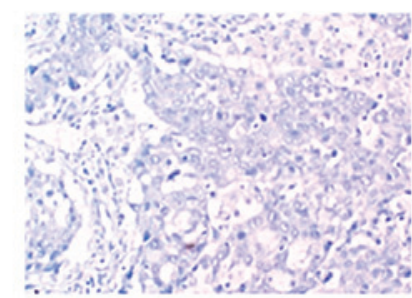

B

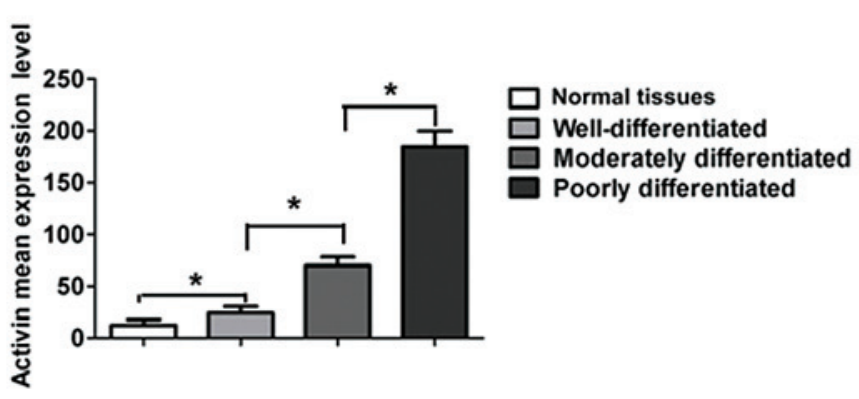

Figure 3. Comparison of activin A expression levels in cancer tissues with various degrees of differentiation. (A) Immunohistochemical analysis and (B) quantitative analysis, revealing the expression of activin A in well-, moderately- and poorly-differentiated cancer tissues.

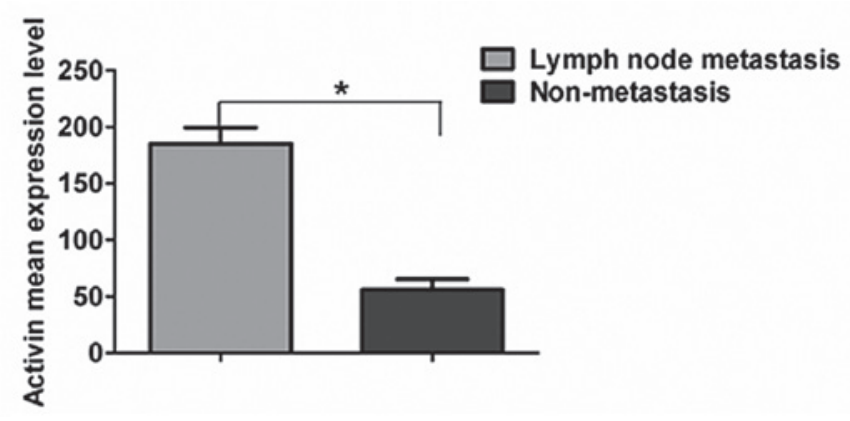

Figure 4. Comparison of activin A expression levels in patients with lymph node metastasis or no metastasis.

moderately-differentiated tumor tissues compared with that in well-differentiated tumors and normal esophageal tissues $(\mathrm{P}<0.05)$. By contrast, activin A expression in poorly-differentiated carcinoma tissues was significantly higher compared with that in moderately-differentiated tumor tissues $(\mathrm{P}<0.05)$; in addition, the expression in well-differentiated tumors was significantly higher compared with the control normal tissues $(\mathrm{P}<0.05)$.

Correlation between activin A and metastasis in esophageal cancer. Among the 57 patients included in the current study, 37 subjects presented lymph node metastasis and the remaining 20 patients presented no metastasis. Patients were divided into two groups according to the presence or absence of lymph node metastasis. The two groups demonstrated no statistically significant differences in the gender and age index, and were considered to be comparable $(\mathrm{P}>0.05)$. The results revealed that the expression of activin A was markedly higher in metastatic esophageal carcinomas compared with tumors without metastasis, and the difference was statistically significant $(\mathrm{P}<0.05$; Fig. 4).
A

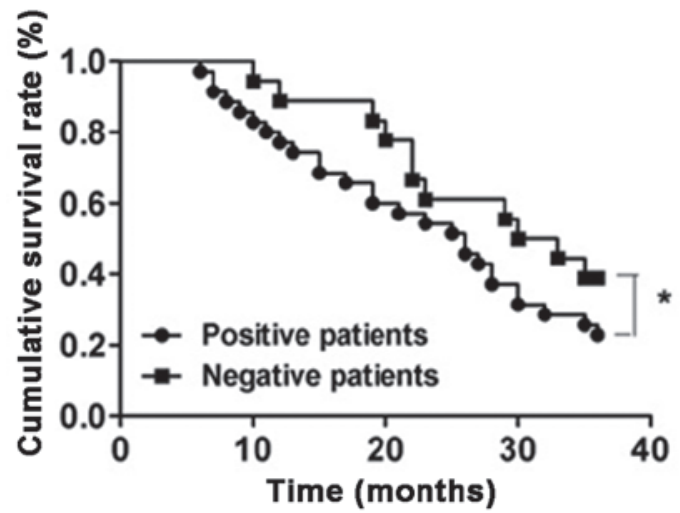

B

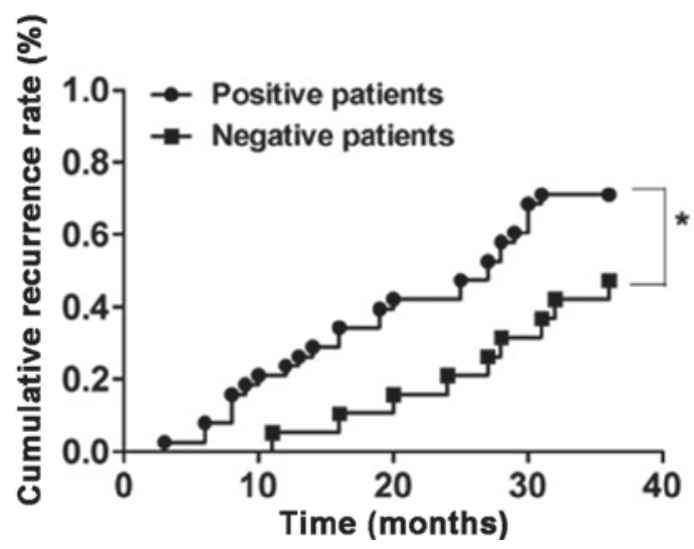

Figure 5. Comparison of postoperative metastasis in activin A-positive and -negative patients. (A) Cumulative survival rate and (B) cumulative recurrence rate in activin A-positive and -negative patients. ${ }^{*} \mathrm{P}<0.05$.

Correlation of activin A with survival and recurrence rates. The 57 patients were divided into two groups according to the evaluation standard for activin A expression in the immunohistochemical assay, which included 38 activin A-positive subjects or 19 activin A-negative subjects. Subsequently, the recurrence and survival rates of each group were analyzed. 
As shown in Fig. 5, the cumulative survival rates at 1,2 and 3 years postoperatively in the positive group were $71.05,50.00$ and $21.05 \%$, respectively, while in the negative group the rates were $84.21,63.16$ and $36.84 \%$, respectively. Patients with a positive expression of activin A had a significantly shorter survival time compared with that of patients with a negative expression of activin A $(\mathrm{P}<0.05)$. Furthermore, the cumulative recurrence rates at 1,2 and 3 years postoperatively in the positive group were found to be $23.68,42.11$ and $71.00 \%$, respectively, while in the negative group the rates were 5.26, 21.05 and $47.37 \%$, respectively. The positive group presented an evidently higher cumulative recurrence rate compared with the negative group $(\mathrm{P}<0.05)$.

\section{Discussion}

Esophageal carcinoma is one of the most common cancer types. A previous study has reported that abnormal expression of activin A was detected in the serum of patients with esophageal carcinoma (19). Therefore, investigating the correlation of the activin A expression with the tumorigenicity and metastasis of human esophageal cancer is essential. However, only a limited number of studies have been conducted on the expression of activin A in esophageal tumor tissues. The present study detected the mRNA and protein expression levels of activin A in esophageal tumor and normal esophageal tissues. The results revealed that the mRNA and protein expression levels of activin A were significantly higher in esophageal cancer compared with those in normal esophageal tissues, which is consistent with the findings of previous studies $(20,21)$.

In addition, the expression of activin $\mathrm{A}$ in esophageal tumor tissues with various degrees of differentiation was investigated. The immunohistochemical assay demonstrated that the protein expression of activin A was the highest in poorly-differentiated tumor tissues and the lowest in well-differentiated esophageal tumor tissues, which revealed an association between activin $\mathrm{A}$ and the differentiation degree of esophageal carcinoma. Thus, a high expression of activin A can be considered to represent a high degree of malignancy, while a low expression of activin A may indicate a benign tumor. Furthermore, analysis of the lymph node metastasis demonstrated that the expression of activin A was higher in esophageal tumors with presence of lymph node metastasis compared with absence of metastasis. This result indicates that activin A may be involved in the metastasis of esophageal cancer, which is consistent with the aforementioned association with the differentiation degree of esophageal carcinoma. Malignant tumors are generally more likely to metastasize, while benign tumors demonstrate few metastases. The results of the present study revealed that activin A plays an important role in tumor metastasis and grade malignancy, which has also been observed in numerous other types of cancer (22-24).

The primary measure for the treatment of esophageal carcinoma is surgery; however, recurrence is observed postoperatively in certain patients and affects the survival of these patients (25). The present study confirmed that patients with high expression of activin A preserved a lower cumulative survival rate and a higher cumulative recurrence rate compared with patients with low expression of activin A. These results suggest that activin A is closely associated with the recurrence and survival of esophageal cancer patients, which may be due to its correlation with the metastasis and malignancy of esophageal tumors.

In conclusion, the present study identified that activin A was abnormally expressed in esophageal carcinoma tissues and correlated with the tumor differentiation, metastasis, recurrence and survival of esophageal carcinoma patients. Therefore, activin A may be a potential molecular marker for the diagnosis and postoperative prognosis of esophageal cancer.

\section{References}

1. Siegel R, Naishadham D and Jemal A: Cancer statistics, 2012. CA Cancer J Clin 62: 10-29, 2012.

2. Jiang L, Zhao X, Meng X and Yu J: Involved field irradiation for the treatment of esophageal cancer: Is it better than elective nodal irradiation? Cancer Lett 357: 69-74, 2015.

3. McK Manson J and Beasley W: A personal perspective on controversies in the surgical management of oesophageal cancer. Ann R Coll Surg Engl 96: 575-578, 2014.

4. Baba Y, Watanabe M, Yoshida N and Baba H: Neoadjuvant treatment for esophageal squamous cell carcinoma. World J Gastrointest Oncol 6: 121-128, 2014.

5. Napier KJ, Scheerer M and Misra S: Esophageal cancer: A Review of epidemiology, pathogenesis, staging workup and treatment modalities. World J Gastrointest Oncol 6: 112-120, 2014.

6. Ling N, Ying SY, Ueno N, Shimasaki S, Esch F, Hotta M and Guillemin R: A homodimer of the beta-subunits of inhibin A stimulates the secretion of pituitary follicle stimulating hormone. Biochem Biophys Res Commun 138: 1129-1137, 1986.

7. Lin HS, Gong JN, Su R, et al: miR-199a-5p inhibits monocyte/ macrophage differentiation by targeting the activin A type $1 \mathrm{~B}$ receptor gene and finally reducing $\mathrm{C} / \mathrm{EBP} \alpha$ expression. J Leukoc Biol 96: 1023-1035, 2014.

8. Kariyawasam HH, Semitekolou M, Robinson DS and Xanthou G: Activin-A: a novel critical regulator of allergic asthma. Clin Exp Allergy 41: 1505-1514, 2011.

9. Maeshima A, Miya M, Mishima K, Yamashita S, Kojima I and Nojima Y: Activin A: autocrine regulator of kidney development and repair. Endocr J 55: 1-9, 2008.

10. Xu J, Oakley J and McGee EA: Stage-specific expression of Smad2 and Smad3 during folliculogenesis. Biol Reprod 66: 1571-1578, 2002

11. Zalzman M, Anker-Kitai L and Efrat S: Differentiation of human liver-derived, insulin-producing cells toward the beta-cell phenotype. Diabetes 54: 2568-2575, 2005.

12. Ferreira MC, Witz CA, Hammes LS, Kirma N, Petraglia F, Schenken RS and Reis FM: Activin A increases invasiveness of endometrial cells in an in vitro model of human peritoneum. Mol Hum Reprod 14: 301-307, 2008.

13. Schwall RH, Robbins K, Jardieu P, Chang L, Lai C and Terrell TG: Activin induces cell death in hepatocytes in vivo and in vitro. Hepatology 18: 347-356, 1993.

14. Thissen JP and Loumaye A: Role of activin A and myostatin in cancer cachexia. Ann Endocrinol (Paris) 74: 79-81, 2013 (In French).

15. Hoda MA, Münzker J, Ghanim B, Schelch K, Klikovits T, Laszlo V, Sahin E, Bedeir A, Lackner A, Dome B, et al: Suppression of activin A signals inhibits growth of malignant pleural mesothelioma cells. Br J Cancer 107: 1978-1986, 2012.

16. Hofland J, van Weerden WM, Steenbergen J, Dits NF, Jenster G and de Jong FH: Activin A stimulates AKR1C3 expression and grow th in human prostate cancer. Endocrinology 153: 5726-5734, 2012.

17. Kim YI, Kim BH, Khang I, Cho BN and Lee HK: Cell growth regulation through apoptosis by activin in human gastric cancer SNU-16 cell lines. Oncol Rep 21: 491-497, 2009.

18. Wang G, Wang L, Sun S, Wu J and Wang Q: Quantitative measurement of serum microRNA-21 expression in relation to breast cancer metastasis in Chinese females. Ann Lab Med 35: 226-232, 2015.

19. Gold E and Risbridger G: Activins and activin antagonists in the prostate and prostate cancer. Mol Cell Endocrinol 359: 107-112, 2012. 
20. Zhang Y, Bao YL, Yang MT, Wu Y, Yu CL, Huang YX, Sun Y, Zheng LH and Li YX: Activin A induces SLC5A8 expression through the Smad3 signaling pathway in human colon cancer RKO cells. Int J Biochem Cell Biol 42: 1964-1972, 2010.

21. de Kretser DM, O'Hehir RE, Hardy CL and Hedger MP: The roles of activin A and its binding protein, follistatin, in inflammation and tissue repair. Mol Cell Endocrinol 359: 101-106, 2012

22. Yoshinaga K, Yamashita K, Mimori K, Tanaka F, Inoue H and Mori M: Activin a causes cancer cell aggressiveness in esophageal squamous cell carcinoma cells. Ann Surg Oncol 15: 96-103, 2008.
23. Yoshinaga K, Mimori K, Yamashita K, Utsunomiya T, Inoue $\mathrm{H}$ and Mori $\mathrm{M}$ : Clinical significance of the expression of activin A in esophageal carcinoma. Int J Oncol 22: 75-80, 2003.

24. Liu SG, Li HC, Zhao BS and Cao F: Expression of activin A in tissue and serum of patients with esophageal squamous cell carcinoma and its clinical significance. Zhonghua Zhong Liu Za Zhi 35: 843-847, 2013 (In Chinese).

25. Zhang C, Wang QT, Liu H, et al: Advancement and prospects of tumor gene therapy. Chin J Cancer 30: 182-188, 2011. 